\title{
CIRCLE-PRESERVING FUNCTIONS OF SPHERES
}

\author{
BY
}

\author{
JOEL GIBBONS AND CARY WEBB
}

\begin{abstract}
Suppose a function of the standard sphere $S^{2}$ into the standard sphere $S^{2+m}, m>0$, sends every circle into a circle but is not a circlepreserving bijection of $S^{2}$. Then the image of the function must lie in a five-point set or, if it contains more than five points, it must lie in a circle together with at most one other point. We prove the local version of this theorem together with a generalization to $n$ dimensions. In the generalization, the significance of 5 is replaced by $2 n+1$. There is also proved a 3-dimensional result in which, compared to the $n$-dimensional theorem, we are allowed to weaken the structure assumed on the image set of the function.
\end{abstract}

1. Introduction. It is a nice application of Schwarz' Lemma that the only analytic circle-preserving functions of the 2-sphere are the nondegenerate Moebius transformations $f(z)=(a z+b) /(c z+d)$. This is not the largest class of circle-preserving functions of the 2-sphere, however. If the class is enlarged to include the continuous but not necessarily analytic functions, then conjugation $f(z)=\bar{z}$ and, in general, functions of the form $f(z)=(a \bar{z}+$ b) $/(c \bar{z}+d)$ must be included.

In geometry, nondegenerate Moebius transformations are called homographies and conjugates of nondegenerate Moebius transformations are called antihomographies. The homographies and antihomographies together form a group. The simplest proofs of which we are aware that the elements of this group are the only circle-preserving bijections of the 2-sphere are found in [3] and [5]. The former is in the strictly geometric style. The latter gives an algebraic treatment.

An inversion is a certain sort of antihomography. It is, up to a translation and dilation, simply $z \rightarrow 1 / \bar{z}$. It is easy to see that the group of homographies and antihomographies is generated by the inversions. This group is commonly called, therefore, the inversive group.

The above mentioned proofs that the inversive group is indeed the set of all circle-preserving bijections of the 2-sphere use strongly the assumption of

Received by the editors March 19, 1977 and, in revised form, May 12, 1977 and December 14, 1977.

AMS (MOS) subject classifications (1970). Primary 50D45, 50B20.

Key words and phrases. Circle, sphere, inversive group.

(c) 1979 American Mathematical Society 0002-9947/79/0000-0052/\$05.25 
bijectivity. To remove this assumption is, roughly speaking, one of the purposes of this paper.

There are several results already available in this direction. In $1937 \mathrm{C}$. Carathéodory [2] showed that if a 1-1 function on an arbitrary region of the 2-sphere maps every circle onto a circle, then that function must be the restriction of an element of the inversive group.

Suppose three circles have a point $p$ in common, any two of them intersect at two points, and $q, r$ are two of the three other points of intersection. In 1967 J. Aczel and M. McKiernan [1], in a generalization of Carathéodory's theorem, showed that if a function of these three circles maps them onto three circles, maps points that are cocircular with $p, q$, or $r$ onto cocircular points, and is $1-1$ on the four points of intersection, then that function must be the restriction of an element of the inversive group.

In 1975 we proved a global generalization of these local results. It was proved that if a function of the 2 -sphere sends every circle into a circle (not necessarily unique) and the image set of this function either contains a set of positive Lebesgue measure or contains at least two circles, then this function is an element of the inversive group. A partial announcement of this appears in [4].

In this paper we obtain a local theorem that generalizes all of the above results. According to the theorem, if a circle-preserving function on an open subset of $S^{2}$ to $S^{2+m}$ possesses six image points no five of which lie on a circle, then that function is an element of the inversive group of $S^{2}$.

The idea of a circle-preserving transformation can be generalized to the $n$-sphere $S^{n}$. In this context, the inversive group is the set of all bijections of $S^{n}$ that preserve $(n-1)$-spheres. (All spheres of lower dimension are, of course, also preserved.) It should be noted that, as in the 2-dimensional case, these inversive groups are made up of products of similarities and inversions.

There are two previously established results of which we are aware that characterize the higher dimensional inversive groups $(n \geqslant 3)$. They are both reported in [6]. The first, due to Moebius and found on p. 310, vol. III of [6], states that every continuous function from an open subset of $S^{3}$ onto an open subset of $S^{3}$ that sends (portions of) 2-spheres to (portions of) 2-spheres must be the restriction of an element of the inversive group. The second, due to Liouville and found on p. 310, vol. III and p. 13, vol. IV of [6], states that every conformal continuous function from an open subset of $S^{n}$ onto an open subset of $S^{n}$ must be the restriction of an element of the inversive group.

Our $n$-dimensional theorem $(n \geqslant 2)$ which is roughly stated at the end of this paragraph (its precise statement is given later as Theorem 3) has no injectivity or continuity hypothesis and no surjectivity hypothesis other than the existence of $2 n+2$ image points. In addition, rather than an assumption 
on $(n-1)$-spheres we need only the hypothesis that circles are sent into circles. The theorem is that if a circle-preserving function from $S^{n}$ to $S^{n+m}$ has at least $2 n+2$ image points distributed regularly (a term to be made precise later) throughout the sphere $S^{n+m}$ then that function must be an element of the inversive group of $S^{n}$.

We emphasize that a function, as we are here using the term, is, except for being single-valued, a completely general correspondence with no connotation of being 1-1, continuous, or having any other usually presumed property. Also, where we say a circle is sent into a circle we do not presuppose its image is more than a single point. This means, of course, that, on the face of it, the image of a circle, if it is one or two points, is actually contained in many circles.

In order to help delimit the problem it is worthwhile to list several sorts of circle-preserving functions of the 2-sphere that are not elements of the inversive group.

(1) Any partition of $S^{2}$ into $n$ nonempty sets induces a circle-preserving function with an $n$ point image lying on a circle.

(2) If $C$ is a circle and $p \notin C$, the function that leaves $C$ point-wise fixed and sends all other points to $p$ is a circle-preserving function whose image is precisely $C \cup\{p\}$. (Actually, it is easy to see how the image can be arranged to be $\{p\}$ together with any subset of $C$.)

(3) Partition the plane into a family of ellipses $e_{a}=\left\{2 x^{2}+y^{2}=a>0\right\}$. Let $\cup A_{r}$ be a partition of the positive reals into countable, dense subsets indexed over the entire set of real numbers and $\infty$. Thinking now of points as being on the Riemann sphere, if $a \in A_{r}$, send all points of $e_{a}$ into $r$. Send 0 to 0 and $\infty$ to $\infty$. Call this map $f$. Let $L$ be the real line, including $\infty$. If $z \in L$, $f^{-1}(z)$ is a countable family of ellipses, dense on the sphere. Therefore $f^{-1}(z)$ meets every circle. So $f$ is circle-preserving, mapping every circle onto the circle $L$.

(4) Think of $S^{2}$ as the Riemann sphere and let $A$ be a dense subset of the equator that is symmetric with respect to the center of the sphere and whose complement is also dense. Let $f$ fix $\infty$. If $x \neq \infty$ lies on a great circle through $\infty$ and a point of $A$, define $f(x)=1$. If $x$ is otherwise, define $f(x)=0$. Then circles not through $\infty$ map to $\{0,1\}$ and circles through $\infty$ map to either $\{1, \infty\}$ or $\{0,1, \infty\}$.

Throughout our discussion, lower case letters will denote points, upper case letters sets of points, subscripts for like objects, and primes for images under the function $f$. The notable exception to these conventions will be when the image $f(C)$ of a circle $C$ is not presumed to be a circle but only contained in a circle. For convenience this latter circle will be denoted $C^{\prime}$ and we will write $f(C) \subseteq C^{\prime}$. 
II. The 2-dimensional case. We intend to prove the following theorem.

TheOREM 1 ("Six PoINT ThEOREM"). Suppose (1) $m$ is a nonnegative integer, (2) $U$ is an open subset of $S^{2}$, (3) $f$ is a function from $U$ to the sphere $S^{2+m}$, (4) $f$ sends a (portion of a) circle into a circle (not assumed to be unique), (5) every circle in $S^{2+m}$ omits at least two points in the image of $f$, and (6) the image of $f$ contains (at least) six points. Then the image of $f$ lies in a 2-sphere and $f$ is the restriction to $U$ of an element of the inversive group of $S^{2}$.

The proof is to reduce the problem to the one solved by Carathéodory. The argument is organized into a number of lemmas. In each lemma the hypotheses of $f$ are as in the theorem except in Lemmas 1.1-1.4 where six points are not required to be contained in the image. (The remaining hypotheses only guarantee the existence of five image points.)

Throughout, whenever we consider a circle $C$ we will really be considering those arcs of $C$ that intersect the open set $U$.

LEMMA 1.1. If $R$ is a connected open region in $U, C^{\prime}$ is a circle, and $f(R) \subseteq C^{\prime}$, then $f$ is constant on $R$.

Proof. We may assume without loss of generality that $R$ is an open disc.

Choose image points $a^{\prime}, b^{\prime} \notin C^{\prime}$. For $z^{\prime} \in C^{\prime}$, let $C_{z}^{\prime}$ be the circle $\left(a^{\prime}, b^{\prime}, z^{\prime}\right)$ and, in case $z^{\prime} \in f(R)$, fix $z \in f^{-1}\left(z^{\prime}\right) \cap R$ and let $C_{z}$ be the circle $(a, b, z)$. For any $z^{\prime} \in C^{\prime}$, let $z_{1}^{\prime}$ be the other point, if it exists, at which $C_{z}^{\prime}$ meets $C^{\prime}$. Call the pair $\left\{z^{\prime}, z_{1}^{\prime}\right\}$ a "distinguished pair." (If $C_{z}^{\prime}$ is tangent to $C^{\prime}$ or if $C_{z}^{\prime}$ and $C^{\prime}$ are not in the same plane, then $z^{\prime}=z_{1}^{\prime}$ and the "pair" is actually a singleton.)

Suppose $f(R)$ equals a distinguished pair $\left\{z^{\prime}, z_{1}^{\prime}\right\}$ and $z^{\prime} \neq z_{1}^{\prime}$. Choose an image point $x^{\prime} \notin C_{z}^{\prime}$. Consider the circles $D^{\prime}=\left(a^{\prime}, x^{\prime}, z^{\prime}\right)$ and $D_{1}^{\prime}=$ $\left(a^{\prime}, x^{\prime}, z_{1}^{\prime}\right)$. Consider also circles of the sort $C_{w}=(a, x, w)$ where $w \in R$. Note $C_{w}^{\prime} \subseteq D^{\prime}$ or $C_{w}^{\prime} \subseteq D_{1}^{\prime}$.

If we let $A_{w}$ be the arc $C_{w} \cap R$, it follows that $f$ is constant on $A_{w}$. So there must be an arc, say $A_{v}$, which maps to a point of $\left\{z^{\prime}, z_{1}^{\prime}\right\}$, say $z^{\prime}$, and that is approximated by a sequence of arcs $\left\{A_{w_{n}}\right\}_{n=1}^{\infty}$, each mapping to $z_{1}^{\prime}$. Hence for every $v \in A_{v}$, every circle transverse to $C_{v}$ at $v$ meets $f^{-1}\left(z^{\prime}\right)$ and $f^{-1}\left(z_{1}^{\prime}\right)$. $(b, v, x)$ is such a circle. Hence the set $\left\{x^{\prime}, b^{\prime}, z^{\prime}, z_{1}^{\prime}\right\}$ lies in a circle, contradicting our choice of $x^{\prime}$. We conclude that either $f$ is constant on $R$ or $f(R)$ is not a distinguished pair $\left\{z^{\prime}, z_{1}^{\prime}\right\}, z^{\prime} \neq z_{1}^{\prime}$.

Suppose now that $f(R)$ meets at least two distinguished pairs. In a fashion similar to the preceding, consider the family of circles $C_{w}=(a, b, w)$ where $w \in R$ and the corresponding family of arcs $A_{w}=C_{w} \cap R$. If $f$ maps two such arcs into intersecting distinguished pairs, they must be the same pair.

Since $R$ is connected and we have supposed $f(R)$ does not lie within only one distinguished pair, there must be an arc $A_{w}-w$ now fixed-mapping to a 
distinguished pair $\left\{z^{\prime}, z_{1}^{\prime}\right\}$ which is approximated by arcs $\left\{A_{w_{n}}\right\}_{n=1}^{\infty}$ each mapping to a distinguished pair disjoint from $\left\{z^{\prime}, z_{1}^{\prime}\right\}$.

A circle through $w$ and transverse to $A_{w}$ meets all but a finite number of the $\operatorname{arcs} A_{w_{n}}$. Hence we may assume all of the arcs $A_{w_{n}}$ map to a single distinguished pair $\left\{y^{\prime}, y_{1}^{\prime}\right\}$ disjoint from $\left\{z^{\prime}, z_{1}^{\prime}\right\}$. In addition, every $x \in R$ lies on a circle through $a$, and one point from each of $A_{w}$ and one of the $\operatorname{arcs} A_{w_{n}}$. Therefore $f(R) \subseteq\left\{y^{\prime}, y_{1}^{\prime}, z^{\prime}, z_{1}^{\prime}\right\}$. There are three cases to consider.

The first case is that, on all but finitely many of the arcs $A_{w_{n}}, f$ is constant, say $f\left(A_{w_{n}}\right)=y^{\prime}$. If $x \in S^{2}-C_{w}$, the circle $(a, w, x)$ is transverse to $A_{w}$ at $w$ and, therefore, maps to a circle through $a^{\prime}, y^{\prime}$ and one point of $\left\{z^{\prime}, z_{1}^{\prime}\right\}$, say the circle is $\left(a^{\prime}, y^{\prime}, z^{\prime}\right)$. Likewise for $b$ replacing $a$. Therefore $S^{2}-C_{w} \subseteq$ $\left\{y^{\prime}, z^{\prime}\right\}$, so the image of $f$ is $C_{w}^{\prime} \cup\left\{y^{\prime}, z^{\prime}\right\}=C_{w}^{\prime} \cup\left\{y^{\prime}\right\}$. But this contradicts our assumption on the image of $f$.

The second case is that $f$ is nonconstant on an infinite number of the arcs $A_{w_{n}}$ and $f^{-1}\left(\left\{y^{\prime}, y_{1}^{\prime}\right\}\right) \cap R$ is not open in $R$. Accordingly, there is $y \in$ $f^{-1}\left(\left\{y^{\prime}, y_{1}^{\prime}\right\}\right) \cap R$, say $y \in f^{-1}\left(y^{\prime}\right)$, such that $y$ is approached by an infinite sequence of arcs, each mapping to $\left\{z^{\prime}, z_{1}^{\prime}\right\}$. We may choose $y_{1} \in R-A_{y}$ such that $f\left(y_{1}\right)=y_{1}^{\prime}$. So $f$ sends the circle $\left(a, y, y_{1}\right)$ into the circle $\left(a^{\prime}, y^{\prime}\right.$, $\left.y_{1}^{\prime}, z^{\prime}\right)$ or the circle $\left(a^{\prime}, y^{\prime}, y_{1}^{\prime}, z_{1}^{\prime}\right)$, again a contradiction.

The last case is that $f$ is nonconstant on an infinite number of the $\operatorname{arcs} A_{w_{n}}$ and $f^{-1}\left(\left\{y^{\prime}, y_{1}^{\prime}\right\}\right) \cap R$ is open. If $S$ is a component of this set then by the first part of our argument $f$ is constant on $S$. Therefore there is an arc meeting the boundary of $S$ to which the first case of the argument applies.

LEMMA 1.2. $f$ cannot map a region into a circle.

Proof. Suppose $R$ is an open disc, $C^{\prime}$ is a circle, and $f(R) \subseteq C^{\prime}$. By Lemma $1.1, f(R)=\left\{z^{\prime}\right\}$.

Let $S$ be the largest connected open region containing $R$ whose image is $a^{\prime}$. Choose two image points $b^{\prime}$ and $c^{\prime}$ that are distinct from $a^{\prime}$. Let $p$ be a point on the boundary of $S$ such that the circle $(b, c, p)$ is not tangent to $S$. Then circles through $b, c$ and points of $S$ contain a neighborhood $N$ of $p$. By Lemma 1.1, $N \subseteq S$. This contradiction establishes Lemma 1.2.

LEMma 1.3. If $C$ and $C^{\prime}$ are circles and $f(C) \subseteq C^{\prime}$ then $f(C)$ misses at most one image point of $C^{\prime}$.

Proof. Suppose $a^{\prime}, b^{\prime} \in C^{\prime}-f(C)$. The family of circles through $a, b$ and points of $C$ contain an open set mapping to $C^{\prime}$. This contradicts Lemma 1.2.

LEMMA 1.4. If $A$ is an arc of a circle, then the image of $A$ under $f$ contains at least three points.

Proof. Let $C$ be a circle meeting $A$ at two points and suppose the image is $A^{\prime}=\left\{a^{\prime}, b^{\prime}\right\}, a^{\prime}$ and $b^{\prime}$ not being necessarily distinct. Choose two points $c$, 
$d \in C \cap U$ that are separated by $A$. This can be done so that $c^{\prime} \neq d^{\prime}$, otherwise there would be a region mapping into a circle, contradicting Lemma 1.2.

Every point of the sphere lies on a circle through $c, d$ and $A$. So $a^{\prime} \neq b^{\prime}$ and the image of $f$ is contained in the necessarily distinct circles $\left(a^{\prime}, c^{\prime}, d^{\prime}\right)$ and $\left(b^{\prime}, c^{\prime}, d^{\prime}\right)$.

Because of our hypothesis on $f$ we are able to choose a fourth image point $e^{\prime}$ on the circle $\left(b^{\prime}, c^{\prime}, d^{\prime}\right)$. A point $e \in f^{-1}\left(e^{\prime}\right)$ may be chosen interior to $C$ by Lemma 1.2. Suppose that $A$ separates $e$ and $d$ relative to the interior of $C$. Then, for the same reason as was used in the preceding paragraph, the entire image of $f$ is contained in the two circles $\left(a^{\prime}, d^{\prime}, e^{\prime}\right)$ and $\left(b^{\prime}, d^{\prime}, e^{\prime}\right)=$ $\left(b^{\prime}, c^{\prime}, d^{\prime}\right)$. But we previously had shown the image to be contained in the two circles $\left(a^{\prime}, c^{\prime}, d^{\prime}\right)$ and $\left(b^{\prime}, c^{\prime}, d^{\prime}\right)$. Therefore the image is exactly the circle $\left(b^{\prime}, c^{\prime}, d^{\prime}\right)$ together with $\left\{a^{\prime}\right\}$, a clear contradiction.

Lemma 1.5. Suppose $C$ and $C^{\prime}$ are circles, $f(C) \subseteq C^{\prime}$, and $C^{\prime}$ contains at least four image points. Then the inverse image in $C$ of any element in $C^{\prime}$ is nowhere dense in $C$.

Proof. Suppose the contrary, $a^{\prime} \in C^{\prime}$, and $A$ is an arc of $C \cap U$ in which $f^{-1}\left(a^{\prime}\right)$ is dense. By Lemma 1.4 we can choose two points, $b, c \in A$ whose images are distinct and different from $a^{\prime}$.

Suppose there were circles $C_{1}^{\prime}$ and $C_{2}^{\prime}$ satisfying (1) $C_{1}^{\prime} \cap C_{2}^{\prime} \cap$ image of $f=\varnothing$, (2) $a^{\prime} \in C_{1}^{\prime}$, (3) $\left\{b^{\prime}, c^{\prime}\right\} \cap C_{2}^{\prime} \neq \varnothing$, and (4) $C_{1}^{\prime}$ and $C_{2}^{\prime}$ each contain three image points, say $C_{1}^{\prime}=\left(a^{\prime}, a_{1}^{\prime}, a_{2}^{\prime}\right)$ and $C_{2}^{\prime}=\left(b^{\prime}, b_{1}^{\prime}, b_{2}^{\prime}\right)$.

Denote by $C_{2}$ the circle $\left(b, b_{1}, b_{2}\right)$ and by $C_{x}$ the circle $\left(x, a_{1}, a_{2}\right)$ for $x \in f^{-1}\left(a^{\prime}\right) \cap A . C_{x} \cap C_{2}=\varnothing$ for all such $x$. However the circles $C_{x}$ are dense in a neighborhood of $b$ and, therefore, must intersect $C_{2}$. So the existence of $C_{1}^{\prime}$ and $C_{2}^{\prime}$ has led to a contradiction. The intent of our proof is to show the existence of $C_{1}^{\prime}$ and $C_{2}^{\prime}$.

Case 1. Relative to some 2-sphere containing $C^{\prime}$ the interior and exterior of $C^{\prime}$ both contain a point in the image of $f$. We can assume the exterior point $\infty$.

Choose four distinct image points $p_{1}^{\prime}, p_{2}^{\prime}, p_{3}^{\prime}, p_{4}^{\prime} \in C^{\prime}$ and assume their order is clockwise around $C^{\prime}$. Let $L_{i}^{\prime}$ be the line through $p_{i}^{\prime}$ and $p_{i+1}^{\prime}$. (Here and in what follows, $i+j$ will stand for its residue modulo 4.) Label the circle through $p_{i+2}^{\prime}$ and $p_{i+3}^{\prime}$ that is tangent to $L_{i}^{\prime}$ within (resp. without) $C^{\prime}$ by $D_{i}^{\prime}$ (resp. $D_{i}^{\prime \prime}$ ). Define $S_{i}$ to be the open region interior to $D_{i}^{\prime}$ and exterior to $D_{i}^{\prime \prime}$. Let $S=\left(\cup_{i=1}^{4} S_{i}\right) \cup\left\{p_{1}^{\prime}, p_{2}^{\prime}, p_{3}^{\prime}, p_{4}^{\prime}\right\}$ and let $Q$ be the quadrilateral with vertices $p_{1}^{\prime}, p_{2}^{\prime}, p_{3}^{\prime}, p_{4}^{\prime}$.

If $p$ is the intersection of the diagonals of $Q$, then $p$ lies on the $S_{i}$ side of $D_{i}^{\prime}$ because $D_{i}^{\prime}$ meets all three sides of the triangle $\left(p, p_{i}^{\prime}, p_{i+1}^{\prime}\right)$. It follows that $S_{i}$ 
contains one of the line segments $\left(p, p_{i+2}^{\prime}\right)$ or $\left(p, p_{i+3}^{\prime}\right)$. (If not, then $D_{i}^{\prime \prime}$ must meet both diagonals of $Q$, but in such case the arc of $D_{i}^{\prime \prime}$ exterior to $C^{\prime}$ lies entirely in the region missing $L_{i}^{\prime}$ that is bounded by the lines $\left(p_{i}^{\prime}, p_{i+2}^{\prime}\right)$ and $\left.\left(p_{i+1}^{\prime}, p_{i+3}^{\prime}\right).\right)$ Now it is clear that $S$ covers at least two of the segments $\left(p, p_{i}^{\prime}\right)$, $i=1,2,3,4$, and that, if just two, they form a diagonal. In any case, $S$ contains a diagonal of $Q$.

Suppose $y^{\prime} \in \cup_{i=1}^{4} S_{i}$, say $y^{\prime} \in S_{1}$. The circle $\left(y^{\prime}, p_{3}^{\prime}, p_{4}^{\prime}\right)$ and the line $\left(p_{1}, p_{2}^{\prime}\right)$ fulfill the conditions of $C_{1}^{\prime}$ and $C_{2}^{\prime}$ for $a^{\prime} \in\left\{p_{1}^{\prime}, p_{2}^{\prime}, p_{3}^{\prime}, p_{4}^{\prime}\right\}$. Therefore, we may assume $\cup_{i=1}^{4} S_{i} \cap$ image of $f=\varnothing$. (So that the interior of one of the diagonals of $Q$ does not meet the image of $f$.)

Choose an image point $q^{\prime}$ within the interior of $C^{\prime}$. (The hypothesis of Case 1 guarantees it.) Suppose the open segment $\left(p_{1}^{\prime}, p_{3}^{\prime}\right)$ is the image-free diagonal of $Q$. Consider the circles $\left(q^{\prime}, p_{2}^{\prime}, p_{4}^{\prime}\right)$ and $\left(q^{\prime}, p_{1}^{\prime}, p_{3}^{\prime}\right)$. These circles contain the diagonals of a quadrilateral with vertices $p_{1}^{\prime}, p_{2}^{\prime}, p_{3}^{\prime}, p_{4}^{\prime}$. (The point $q^{\prime}$ may be thought to play the role of $\infty$.) The same argument as before now shows that either the open $\operatorname{arc}\left(p_{1}^{\prime}, p_{3}^{\prime}\right)$ or the open $\operatorname{arc}\left(p_{2}^{\prime}, p_{4}^{\prime}\right)$-neither containing $q^{\prime}$, of course-is image-free. If it is the former, the circles $\left(p_{1}^{\prime}, p_{3}^{\prime}, q^{\prime}\right)$ and $\left(p_{2}^{\prime}, p_{4}^{\prime}, \infty\right)$ are the circles $C_{1}^{\prime}$ and $C_{2}^{\prime}$. If it is the latter, the circles $\left(p_{1}^{\prime}, p_{3}^{\prime}, \infty\right)$ and $\left(p_{2}^{\prime}, p_{4}^{\prime}, q^{\prime}\right)$ are the circles $C_{1}^{\prime}$ and $C_{2}^{\prime}$. The only way the appropriate pair of circles could not fulfill all the requirements of $C_{1}^{\prime}$ and $C_{2}^{\prime}$ is that their point $r^{\prime}$ of intersection within $C^{\prime}$ is an image point. But, recalling $r^{\prime} \notin \cup_{i=1}^{4} S_{i}$, if this were true the circle $\left(r^{\prime}, p_{1}^{\prime}, p_{2}^{\prime}\right)$ would meet the line $\left(p_{3}^{\prime}, p_{4}^{\prime}\right)$-speaking now of the case when $C_{1}^{\prime}$ and $C_{2}^{\prime}$ are $\left(p_{1}^{\prime}, p_{3}^{\prime}, q^{\prime}\right)$ and $\left(p_{2}^{\prime}, p_{4}^{\prime}, \infty\right)$. This clearly cannot occur. (A similar statement can be made concerning the other possibility for $C_{1}^{\prime}$ and $C_{2}^{\prime}$.)

Case 2. The image of $f$ on a 2-sphere containing $C^{\prime}$ consists of points on $C^{\prime}$ together with at least two other points and is contained in $C^{\prime}$ and its interior.

Let $p_{1}^{\prime}, p_{2}^{\prime}, p_{3}^{\prime}, p_{4}^{\prime}$ be as before and choose an image point $p^{\prime}$ within $C^{\prime}$.

For a pair of adjacent points $p_{i}^{\prime}$ and $p_{i+1}^{\prime}$, consider the circle $E_{i}^{\prime}=$ $\left(p^{\prime}, p_{i}^{\prime}, p_{i+1}^{\prime}\right)$. Let $F_{i}^{\prime}$ be the circle through $p_{i+2}^{\prime}$ and $p_{i+3}^{\prime}$ that is tangent to $E_{i}^{\prime}$ inside $C^{\prime}$. Let $T_{i}$ be the points interior to both $F_{i}^{\prime}$ and $C^{\prime}$. The complement within $C^{\prime}$ of $T=\cup_{i=1}^{4} T_{i}$ is either $\left\{p^{\prime}\right\}$ or a quadrilateral $Q_{1}$ with sides arcs of the circles $E_{1}^{\prime}, E_{2}^{\prime}, E_{3}^{\prime}, E_{4}^{\prime}$. Note that $p^{\prime} \in Q_{1}$. Also note that $T$ contains no image point or else we would clearly have circles $C_{1}^{\prime}$ and $C_{2}^{\prime}$.

Suppose $Q_{n}$ is a quadrilateral containing $p^{\prime}$ with sides arcs of circles. Consider the largest circle through $Q_{n}, p_{i+2}^{\prime}$, and $p_{i+3}^{\prime}$ that does not meet $E_{i}^{\prime}$ on the interior of $Q_{n}$. These four circles determine the point $\left\{p^{\prime}\right\}$ or a quadrilateral $Q_{n+1}$ properly nested within $Q_{n}$ in such a fashion that $\cap_{n=1}^{\infty} Q_{n}=$ $\left\{p^{\prime}\right\}$.

Note that there is no image point other than $p^{\prime}$ interior to $C^{\prime}$-otherwise the circles $C_{1}^{\prime}$ and $C_{2}^{\prime}$ would exist. Hence the image of $f$ on the 2-sphere in 
question is contained in $C^{\prime} \cup\left\{p^{\prime}\right\}$. Now let $q^{\prime}$ be an image point off of this 2-sphere, let $C_{1}^{\prime}$ be the circle $\left(p_{1}^{\prime}, p_{2}^{\prime}, q^{\prime}\right)$, and let $C_{2}^{\prime}$ be the circle $\left(p_{3}^{\prime}, p_{4}^{\prime}, p^{\prime}\right)$.

Case 3. The image of $f$ on a 2-sphere containing $C^{\prime}$ consists of the image points on $C^{\prime}$ together with one other point $p^{\prime}$.

In such case, let $q^{\prime}$ be an image point off of this 2-sphere, let $C_{1}^{\prime}$ be the circle $\left(p_{1}^{\prime}, p_{2}^{\prime}, q^{\prime}\right)$, and let $C_{2}^{\prime}$ be the circle $\left(p_{3}^{\prime}, p_{4}^{\prime}, p^{\prime}\right)$. This completes the proof of Lemma 1.5.

LEMMA 1.6. If the circle $C^{\prime}$ meets the image of $f$ in at least four points then its inverse image is the intersection with $U$ of a unique circle.

Proof. Suppose $C$ is a circle and $f(C) \subseteq C^{\prime}$. If $a \in f^{-1}\left(C^{\prime}\right)-C$, choose $b \in C \cap U$ and an arc $A$ of $C \cap U$ so that $A^{\prime}$ contains neither $a^{\prime}$ nor $b^{\prime}$ (Lemma 1.5). Then the intersection with $U$ of every circle $(a, b, c)$ for $c \in A$ maps to $C^{\prime}$. Since this family of circles covers an open set we have contradicted Lemma 1.2.

LEMMA 1.7. If the image of $f$ contains at least six points, then the image of every circle contains at least four points.

Proof. Suppose, on the contrary, that the image of a circle $C=\left(p_{1}, p_{2}, p_{3}\right)$ is precisely the set $\left\{p_{1}^{\prime}, p_{2}^{\prime}, p_{3}^{\prime}\right\}$. If we could construct circles $C_{1}^{\prime}$ and $C_{2}^{\prime}$ as defined in the proof of Lemma 1.5 then we would be led to a contradiction for exactly the same reason as in Lemma 1.5. So we may assume if $C_{1}^{\prime}$ and $C_{2}^{\prime}$ are circles, $p_{1}^{\prime} \in C_{1}^{\prime},\left\{p_{2}^{\prime}, p_{3}^{\prime}\right\} \cap C_{2}^{\prime} \neq \varnothing$, and $C_{1}^{\prime}$ and $C_{2}^{\prime}$ each meet the image of $f$ in at least three points, then $C_{1}^{\prime} \cap C_{2}^{\prime}$ contains an image point.

Since the image set of $f$ contains at least six points, the complement of $C^{\prime}$ contains (at least) three image points $a^{\prime}, b^{\prime}, c^{\prime}$. Let $C_{1}^{\prime}$ be the circle $\left(p_{1}^{\prime}, a^{\prime}, b^{\prime}\right)$ and $C_{2}^{\prime}$ the circle $\left(p_{2}^{\prime}, p_{3}^{\prime}, c^{\prime}\right)$. Denote by $d^{\prime}$ an image point in $C_{1}^{\prime} \cap C_{2}^{\prime}$.

If $d^{\prime} \neq c^{\prime}, C_{2}^{\prime}$ is a circle meeting the image of $f$ at four points. So $f^{-1}\left(C_{2}^{\prime}\right)=C_{2} \cap U$ is the arc of a unique circle (Lemma 1.6). In such case $f^{-1}\left(p_{2}^{\prime}\right)$ and $f^{-1}\left(p_{3}^{\prime}\right)$ are singletons-otherwise there would exist $p_{4} \in C$ such that $p_{4}^{\prime}=p_{2}^{\prime}$ (or, $\left.p_{4}^{\prime}=p_{3}^{\prime}\right)$ so the circle $\left(c, p_{3}, p_{4}\right) \neq C_{2}$ would also map to $C_{2}^{\prime}$. But this situation clearly contradicts Lemma 1.4. Thus $d^{\prime}=c^{\prime}$.

Consider now the circle $\left(p_{1}^{\prime}, a^{\prime}, b^{\prime}, c^{\prime}\right)$. Since this circle contains four points we are again led to a contradiction for the same reason. The lemma is therefore established.

An immediate consequence of Lemmas 1.6 and 1.7 is

LEMMA 1.8. If the image of $f$ contains at least six points, then the inverse image of every circle is (the arc of) a unique circle.

To prove $f$ is $1-1$ is now easy.

Suppose $a \neq b$ and $a^{\prime}=b^{\prime}$. Choose points $c$ and $d$ whose images are 
distinct and different from $a^{\prime}$. By Lemma $1.8, b$ lies on the circle $(a, c, d)$. Choose $e^{\prime} \notin\left(a^{\prime}, c^{\prime}, d^{\prime}\right)$. Then the circles $(a, c, e)$ and $(b, c, e)$ are distinct and map to $\left(a^{\prime}, c^{\prime}, e^{\prime}\right)$. This contradicts Lemma 1.8. We have shown, therefore, that $f$ is $1-1$.

In order to apply Carathéodory's result, we need to show that the image of a circle $C$ in $U$ is an (entire) circle. It clearly suffices to suppose $U$ is a disc.

By Lemma 1.7, $f(C)$ contains at least four points. Suppose $C^{\prime}$ is the circle determined by $f(C)$. By Lemma $1.6, f^{-1}\left(C^{\prime}\right)$ is the unique circle $C$. (Another way of seeing this is to use Lemma 1.4 to prove that $f(C)$ contains at least three points and then apply Lemma 1.8.)

Suppose $z \in C^{\prime}-f(C)$. Choose $y \in U-C$, two image points $x_{1}^{\prime}, x_{2}^{\prime} \in$ $C^{\prime}$, and let $C_{1}^{\prime}$ (resp. $\left.C_{2}^{\prime}\right)$ be the circle $\left(x_{1}^{\prime}, y^{\prime}, z\right)$ (resp. $\left.\left(x_{2}^{\prime}, y^{\prime}, z\right)\right)$. By Lemma 1.6 or Lemma $1.8, f^{-1}\left(C_{1}^{\prime}\right)=C_{1}$ and $f^{-1}\left(C_{2}^{\prime}\right)=C_{2}$ are unique circles. Since $f$ is $1-1, C_{1}$ and $C_{2}$ are tangent to $C$ at $x_{1}$ and $x_{2}$, respectively. This means $f(C)$ misses at most the one point $z$ of $C^{\prime}$.

Clearly we could have chosen $x_{1}^{\prime}$ and $x_{2}^{\prime}$ so that $C_{1} \cap C_{2}=\{y, w\} \subseteq U$, where $y \neq w$. Since neither $y$ nor $w$ lies on $C$, this immediately leads to a contradiction because $f$ is $1-1$ and $z \in C^{\prime} \cap C_{1}^{\prime} \cap C_{2}^{\prime}$. This establishes that circles in $U$ do indeed map onto circles.

In order to be able to apply Carathéodory's theorem and complete the proof of Theorem 1 it only remains to show that the image of $f$ is contained in a 2-sphere.

Choose a circle $C$ and a point $y \notin C . f(C)=C^{\prime}$ is a circle and $y^{\prime} \notin C^{\prime}$. Denote the 2-sphere determined by $C^{\prime}$ and $y^{\prime}, S^{\prime}$.

If $x \in U-(C \cup\{y\})$, let $C_{1}$ be a circle through $x, y$ and two points of $C$. $f$ maps $C_{1}$ into $S^{\prime}$. Hence $x \in S^{\prime}$. The image of $f$ is, therefore, contained within $S^{\prime}$. The proof of Theorem 1 is now complete.

RemarK. Example (2) in the Introduction shows that in the Six Point Theorem the assumption every circle misses at least two image points cannot be relaxed. Therefore the only possible (2-dimensional) improvement of Theorem 1 would be to lower the number of required image points from six to five.

Whether or not this reduction can be made is unknown. In light of Lemma 1.4 , however, the existence of a circle-preserving function whose image is exactly five points, no four of which lie on a circle, is equivalent to the existence of a partition of the 2 -sphere into five dense subsets in such a way that every arc of every circle meets exactly three of the partitioning sets. In this regard, it may be interesting to note that there is a countable dense subset of $S^{2}$ upon which a circle-preserving function exists whose image is five points. This can be shown by the Baire Category Theorem. Whether this suggests the truth or negation of the Five Point Theorem is unclear to us. 
III. A 3-dimensional theorem. In this section we intend to prove the following theorem.

Theorem 2 (The Eight Point Theorem). Suppose (1) $m$ is a nonnegative integer, (2) $U$ is an open subset of the 3-sphere $S^{3}$, (3) $f$ is a function from $U$ into $S^{3+m}$, (4) $f$ sends a (portion of a) circle into a circle (not presumed to be unique), (5) every 2-sphere in $S^{3+m}$ omits at least three points in the image of $f$, and (6) the image of $f$ contains (at least) eight points. Then, the image of $f$ is contained in a 3-sphere and $f$ is the restriction to $U$ of an element of the inversive group of $S^{3}$.

In the next section where an $n$-dimensional theorem is proved there is another 3-dimensional eight point theorem. The virtue of the present theorem compared to this later one is that no structure is assumed upon the range space whereas in the later one six of the image points are presumed to lie on the image of a 2-sphere. On the other hand, this theorem is not a generalization of the next because of assumption (5).

The proof is to reduce the problem to the one solved by Moebius. As with the Six Point Theorem, the argument is structured into a sequence of lemmas. In contrast to the 2-dimensional case, all the assumptions on $f$ are required from the outset.

LEMMA 2.1. $f$ maps every (portion of a) 2-sphere into a 2-sphere.

PROOF. If such is not true we know from Theorem 1 that there is a (portion of a) 2-sphere $S$ with a five-point image $X=\left\{x_{1}^{\prime}, x_{2}^{\prime}, x_{3}^{\prime}, x_{4}^{\prime}, x_{5}^{\prime}\right\}$ not lying on a 2-sphere.

Let $y_{1}^{\prime}, y_{2}^{\prime}, y_{3}^{\prime} \notin X$ be three image points and denote by $C$ the circle through $y_{1}, y_{2}, y_{3}$. For $x \in S$, let $S_{x}$ be the 2-sphere through $x$ and $C$ and denote $C_{x}=S \cap S_{x}$. Assume that $x$ is chosen so that $U$ contains an arc of $C_{x}$. Except for at most two such $x$ 's, $C_{x}$ is a (nondegenerate) circle and $f\left(C_{x}\right)$ is a 3-point set (Lemma 1.4). Thus $f\left(S_{x} \cap U\right)$ contains at least six points. If $f\left(S_{x} \cap U\right)$ were a set satisfying the hypotheses of Theorem 1 , then $f$ restricted to $C_{x}$ would be 1-1, a contradiction. Thus for a given $x$ such that $C_{x}$ is not a point, we may assume $f\left(C_{x}\right)=\left\{x_{1}^{\prime}, x_{2}^{\prime}, x_{3}^{\prime}\right\}$ and there is a circle containing a 5-point subset of $\left\{x_{1}^{\prime}, x_{2}^{\prime}, x_{3}^{\prime}, y_{1}^{\prime}, y_{2}^{\prime}, y_{3}^{\prime}\right\}$.

There are three cases to consider.

Case 1. There are two image points $y_{1}^{\prime}, y_{2}^{\prime}$ not contained in any of the five 2-spheres $S_{1}^{\prime}, S_{2}^{\prime}, S_{3}^{\prime}, S_{4}^{\prime}, S_{5}^{\prime}$ determined by the points of $X$. In this case, fix $y_{1}^{\prime}$ and $y_{2}^{\prime}$ and let $y_{3}^{\prime}$ be any eighth image point. By what we have said above, either (a) $y_{1}^{\prime}$ or $y_{2}^{\prime}$ lies on a circle with three elements of $X$, a contradiction, or (b) $y_{1}^{\prime}, y_{2}^{\prime}, y_{3}^{\prime}$ and two elements of $X$ lie on a circle, also a contradiction because the entire image of $f$ would then be contained in a 2-sphere together with two additional points. 
Case 2. The image of $f$ is contained in $S_{1}^{\prime} \cup S_{2}^{\prime} \cup S_{3}^{\prime} \cup S_{4}^{\prime} \cup S_{5}^{\prime} \cup\left\{y^{\prime}\right\}$. Choose $y_{1}^{\prime}, y_{2}^{\prime} \notin X$ and $x$ so that $C_{x}$ is a nontrivial (arc of a) circle relative to the circle $\left(y_{1}^{\prime}, y_{2}^{\prime}, y^{\prime}\right)$. Since $y^{\prime} \notin S_{i}, i=1, \ldots, 5$, there must be a circle containing $y_{1}^{\prime}, y_{2}^{\prime}$ and three points of $X$. Suppose these five points are $x_{1}^{\prime}, x_{2}^{\prime}$, $x_{3}^{\prime}, y_{1}^{\prime}, y_{2}^{\prime}$.

Pick $y_{3}^{\prime} \notin\left\{y_{1}^{\prime}, y_{2}^{\prime}, y^{\prime}\right\} \cup X$ so that $y_{3}^{\prime}$ is not on the sphere $\left(x_{1}^{\prime}, x_{2}^{\prime}, x_{3}^{\prime}, x_{4}^{\prime}, y_{1}^{\prime}, y_{2}^{\prime}\right)$. We can do this because we have accounted for only two of the image points lying off this sphere. Adjust, if necessary the choice of $x$, so that $C_{x}$ relative to the circle $\left(y_{1}^{\prime}, y_{2}^{\prime}, y_{3}^{\prime}\right)$ is a nontrivial arc.

Suppose $y_{3}^{\prime}$ is on $S_{3}=\left(x_{1}^{\prime}, x_{2}^{\prime}, x_{4}^{\prime}, x_{5}^{\prime}\right)$. Since the circle $\left(y_{1}^{\prime}, y_{2}^{\prime}, y_{3}^{\prime}\right)$ misses the set $\left\{x_{1}^{\prime}, x_{2}^{\prime}, x_{3}^{\prime}, x_{4}^{\prime}\right\}$, two elements of $\left\{y_{1}^{\prime}, y_{2}^{\prime}, y_{3}^{\prime}\right\}$ must lie on a circle with three elements of $X$. (a) If these two elements are $y_{1}^{\prime}, y_{2}^{\prime}$ then the three elements of $X$ must be $x_{1}^{\prime}, x_{2}^{\prime}, x_{3}^{\prime}$. (b) If these two elements are $y_{1}^{\prime}, y_{3}^{\prime}$ then one of the elements of $X$ must be $x_{5}^{\prime}$. This leads to a contradiction because $y_{1}^{\prime} \notin S_{3}$. (c) If these two elements are $y_{2}^{\prime}, y_{3}^{\prime}$ then one of the elements must again be $x_{5}^{\prime}$ which leads to a contradiction for the same reason.

Since (a) must hold and any point of $S$ lies on such a circle $C_{x}$, we have shown all of $S$ maps to the set $\left\{x_{1}^{\prime}, x_{2}^{\prime}, x_{3}^{\prime}\right\}$, a manifest contradiction.

Case 3. The image of $f$ is contained in $\cup_{i=1}^{5} S_{i}$. As before choose $y_{1}^{\prime}, y_{2}^{\prime}$, $y_{3}^{\prime} \notin X$. If three elements of $X$ and two elements of $\left\{y_{1}^{\prime}, y_{2}^{\prime}, y_{3}^{\prime}\right\}$ lie on a circle then we have a contradiction for exactly the same reasons as in Case 2. (The importance of $y^{\prime}$ in Case 2 was to guarantee that this is true.) If $\left\{x_{1}^{\prime}, x_{2}^{\prime}, y_{1}^{\prime}\right.$, $\left.y_{2}^{\prime}, y_{3}^{\prime}\right\}$ are contained in a circle then this circle must be contained in one of the $S_{i}$ 's, for otherwise any two of the five points would lie on a different $S_{i}$ which would imply the existence of more than five $S_{i}$ 's.

Suppose $\left\{x_{1}^{\prime}, x_{2}^{\prime}, y_{1}^{\prime}, y_{2}^{\prime}, y_{3}^{\prime}\right\} \subseteq S_{5}^{\prime}$. Choose $y_{4}^{\prime} \notin S_{5}^{\prime}$. By the argument at the beginning of the lemma, either three elements of $X$ and two of $\left\{y_{1}^{\prime}, y_{2}^{\prime}, y_{4}^{\prime}\right\}$ or two elements of $X$ and all of $\left\{y_{1}^{\prime}, y_{2}^{\prime}, y_{4}^{\prime}\right\}$ lie on a circle. The former is not possible because such a circle intersects $S_{5}^{\prime}$ in at least one point not in $X$. The latter is not possible either because the circle $\left(y_{1}^{\prime}, y_{2}^{\prime}, y_{4}^{\prime}\right)$ intersects $S_{5}^{\prime}$ at $\left\{y_{1}^{\prime}, y_{2}^{\prime}\right\}$ so can contain at most $x_{5}^{\prime}$.

LEMMA 2.2. The image of (a portion of) some 2-sphere either contains six points no five of which lie on a circle or the intersection of $U$ and a 2-sphere is mapped by $f$ into the union of a circle and a point.

Proof. If there is a 2-sphere with such a 6-point image then by the argument in the last two paragraphs of the proof of Theorem $2, f$ is a bijection. Otherwise, either the image of every 2-sphere is contained in the union of a circle and a point, or there is a 2-sphere whose image is a 5-point set no four points of which lie on a circle. If the latter is true, $S$ is the 2-sphere, and $y_{1}^{\prime}, y_{2}^{\prime}, y_{3}^{\prime} \notin S^{\prime}$, then the argument in paragraph two of the proof 
of Lemma 2.1 shows $y_{1}^{\prime}, y_{2}^{\prime}, y_{3}^{\prime}$ and two points of $X$, say $x_{1}^{\prime}$ and $x_{2}^{\prime}$, lie on a circle $C^{\prime}$.

There must exist a fourth point $y_{4}^{\prime} \notin S^{\prime}$ and $y_{4}^{\prime} \notin C^{\prime}$ because $C^{\prime}$ contains all but three of the points thus far accounted for. By the same argument as above $y_{1}^{\prime}, y_{2}^{\prime}, y_{4}^{\prime}$ lie on a circle $C^{\prime \prime} \neq C^{\prime}$ with two elements of $X$. These two elements of $X$ must be distinct from $x_{1}^{\prime}, x_{2}^{\prime}$. Say they are $x_{3}^{\prime}, x_{4}^{\prime}$.

Again by the same argument, $y_{1}^{\prime}, y_{3}^{\prime}, y_{4}^{\prime}$ must lie on a circle $C^{\prime \prime \prime}, C^{\prime \prime \prime} \neq C^{\prime}$ and $C^{\prime \prime \prime} \neq C^{\prime \prime}$, that contains two points of $X$ distinct from $x_{1}^{\prime}, x_{2}^{\prime}, x_{3}^{\prime}, x_{4}^{\prime}$. But only $x_{5}^{\prime}$ remains. This contradiction establishes the lemma.

REMARK. In light of sentence one of the proof it will be routine for us to assume that the image of a 2 -sphere is contained in the union of a circle and a point.

LemMa 2.3. Suppose $B$ is an open ball in $U, S^{\prime}$ is a 2-sphere and $f(B) \subseteq S^{\prime}$. Then $f$ is constant on $B$.

Proof. Fix image points $y_{1}^{\prime}, y_{2}^{\prime}, y_{3}^{\prime} \notin S^{\prime}$ and let $C$ be the circle through $y_{1}$, $y_{2}, y_{3}$. If $x \in B$ and $x \notin C$, let $S_{x}$ be the 2-sphere determined by $x$ and $C$. Define $D_{x}=S_{x} \cap B$. Then $f\left(D_{x}\right) \subseteq S_{x}^{\prime} \cap S^{\prime}=C_{x}^{\prime}$.

Case 1. $C^{\prime} \cap S^{\prime} \cap f(B)=\varnothing$. Then $C \cap B=\varnothing$ and the two circles $C_{x_{1}}^{\prime}$ and $C_{x_{2}}^{\prime}$ are either identical or disjoint.

If $f\left(D_{x}\right)$ contains three points for some $x$, then $S_{x}$ satisfies the conditions of the Six Point Theorem. Hence $f$ restricted to $S_{x}$ is a bijection and, according to the last two paragraphs of this section, Theorem 2 is proved. If $f\left(D_{x}\right)$ is two points and $f\left(S_{x}\right)$ is not contained in the union of a circle and a point, by Lemma 2.2 and Theorem 1 we are again done. If $f\left(D_{x}\right)$ is two points, say $x_{1}^{\prime}$ and $x_{2}^{\prime}$, and $y_{1}^{\prime}, y_{2}^{\prime}, x_{1}^{\prime}, x_{2}^{\prime}$ are contained in a circle, then $x_{1}^{\prime}$ is not contained in either of the circles $\left(x_{2}^{\prime}, y_{1}^{\prime}, y_{3}^{\prime}\right)$ and $\left(x_{2}^{\prime}, y_{2}^{\prime}, y_{3}^{\prime}\right)$. Thus $f$ is constant on the arcs $D_{x} \cap\left(x_{2}, y_{1}, y_{3}\right)$ and $D_{x} \cap\left(x_{2}, y_{2}, y_{3}\right)$. But this implies $f\left(D_{x}\right)=\left\{x_{2}^{\prime}\right\}$. So we may assume $f$ is constant on every $D_{x}$.

If $f$ is not constant on all of $B$ there is a $D_{x_{0}}$ and a sequence $\left\{D_{x_{n}}\right\}_{n=1}^{\infty}$ such that $D_{x_{n}} \rightarrow D_{x_{0}}$ and $x_{n}^{\prime} \neq x_{0}^{\prime}$ for every $n=1,2, \ldots$ If $x_{1}^{\prime} \neq x_{2}^{\prime}, x_{n}^{\prime}=x_{1}^{\prime}$ and $x_{m}^{\prime}=x_{2}^{\prime}$ for infinitely many $n$ and $m$, choose $x_{1}$ and $x_{2}$ so that $x_{0}, x_{1}, x_{2}$ do not lie on a circle. For any $x \in U-B$, at least one of the circles $\left(x, x_{0}, x_{1}\right)$ and $\left(x, x_{0}, x_{2}\right)$ is transverse to $D_{0}$ at $x_{0}$. So at least one of these circles meets infinitely many $D_{x_{n}}$. Hence $x^{\prime}$ lies on the circle $\left(x_{0}^{\prime}, x_{1}^{\prime}, x_{2}^{\prime}\right) \subseteq S^{\prime}$. This means $f(U) \subseteq S^{\prime}$, a contradiction.

If, on the other hand, $x_{n}^{\prime}=x_{1}^{\prime}$ for all sufficiently large $n$, for any $x \in U-$ $S_{x_{0}}$, the circle $\left(x, x_{0}, y_{1}\right)$ is transverse to $D_{x_{0}}$ at $x_{0}$. Hence $x^{\prime}$ lies on the circle $\left(x_{0}^{\prime}, x_{1}^{\prime}, y_{1}^{\prime}\right)$. But this is true for $y_{2}$ replacing $y_{1}$. So $f(U) \subseteq S_{x_{0}}^{\prime} \cup\left\{x_{0}^{\prime}, x_{1}^{\prime}\right\}$, a contradiction. We have proved that in Case $1, f$ is constant on $B$.

Case 2. $C^{\prime} \cap S^{\prime} \cap f(B) \neq \varnothing$. Suppose $C^{\prime} \cap S^{\prime}=\left\{x_{1}^{\prime}, x_{2}^{\prime}\right\}$ where $x_{1} \in B$ 
and $x_{1}^{\prime}$ and $x_{2}^{\prime}$ are not necessarily distinct. (It may, of course, also be true that $x_{2} \in B$.)

Case 2a. $f(B) \subseteq\left\{x_{1}^{\prime}, x_{2}^{\prime}\right\}$. If the image point $z_{1}^{\prime} \notin C^{\prime}$, let $S_{1}^{\prime}$ be the sphere determined by $C^{\prime}$ and $z_{1}^{\prime}$. Choose an image point $z_{2}^{\prime} \notin S_{1}^{\prime}$, and let $S_{2}^{\prime}$ be the sphere through $x_{1}^{\prime}, y_{1}^{\prime}, z_{1}^{\prime}, z_{2}^{\prime}$. Note that $x_{2}^{\prime} \notin S_{2}^{\prime}$. In the same fashion we can find a sphere $S_{3}^{\prime}$ such that $x_{2}^{\prime} \in S_{3}^{\prime}$ but $x_{1}^{\prime} \notin S_{3}^{\prime}$.

If $x \in B$, the sphere determined by $x, y_{1}, z_{1}, z_{2}$ maps to either $S_{2}^{\prime}$ or $S_{3}^{\prime}$. Hence $f$ is constant on the intersection of this sphere with $B$. But now the last part of the argument in Case 1 applies.

Case 2b. $f(B) \underline{E}\left\{x_{1}^{\prime}, x_{2}^{\prime}\right\}$. Suppose $x_{3}^{\prime} \in f(B)-\left\{x_{1}^{\prime}, x_{2}^{\prime}\right\}$. We may as well assume $f$ is not a bijection on $S_{x_{3}}$ by the last two paragraphs of the proof of Theorem 2. So $f\left(S_{x_{3}}\right) \subseteq C^{\prime} \cup\left\{x_{3}^{\prime}\right\}$ (Lemma 2.2). Thus $f\left(D_{x_{3}}\right) \subseteq\left\{x_{1}^{\prime}, x_{2}^{\prime}, x_{3}^{\prime}\right\}$. It must be true that $f$ must be constant and equal to $x_{3}^{\prime}$ on the arcs that are the intersections of $D_{x_{3}}$ with the circles through $x_{3}, y_{1}, y_{2}$ and $x_{3}, y_{1}, y_{3}$. This implies $f$ is constant on $D_{x_{3}}$. So we have two sorts of $D_{x}$ 's: those that map onto $\left\{x_{1}^{\prime}, x_{2}^{\prime}\right\}$ and those on which $f$ is constant. If $f$ is constant on all $D_{x}$ 's the last part of the argument in Case 1 applies. So we may as well assume the more general case.

If there is a $D_{x}$ that maps to a singleton and this $D_{x}$ is approximated by a sequence of other $D_{x}$ 's which map to singletons distinct from the first, the last part of the argument in Case 1 applies. So we are left with the possibility that every $D_{x}$ sufficiently close to a given $D_{x_{0}}$ maps onto $\left\{x_{1}^{\prime}, x_{2}^{\prime}\right\}$ and that $f\left(D_{x_{0}}\right)=\left\{x_{3}^{\prime}\right\}$.

Let $B_{1}$ be an open ball contained in this family of $D_{x}$ 's, $x \neq x_{0}$. Then $f\left(B_{1}\right)=\left\{x_{1}^{\prime}, x_{2}^{\prime}\right\}$. But now Case $2 \mathrm{a}$ implies $f$ is constant on $B_{1}$, a contradiction. This completes the proof of Lemma 2.3.

LEMMA 2.4. Suppose B is an open ball in $U$. Then $f(B)$ cannot be contained in a 2-sphere.

Proof. If $f(B)$ were contained in a 2-sphere, Lemma 2.3 tells us that $f$ is constant on $B$, say $f(B)=\left\{x^{\prime}\right\}$. Clearly there is a 2-sphere $S^{\prime}$ determined by four image points $y_{1}^{\prime}, y_{2}^{\prime}, y_{3}^{\prime}, y_{4}^{\prime}$ such that $x^{\prime} \notin S^{\prime}$. Let $A$ be the set $\{z: z$ lies on some 2-sphere through $x_{1}, x_{2}, y_{4}$ and $B$ ). ( $A$ resembles a thickened 2-sphere pinched along the circle $\left(x_{1}, x_{2}, y_{4}\right)$.) $B$ lies in one of the components of the interior of $A$.

$f(A \cap U)$ is contained in the 2-sphere $\left(x^{\prime}, x_{1}^{\prime}, x_{2}^{\prime}, y_{4}^{\prime}\right)$ because of Lemma 2.1. Lemma 2.3 says $f$ is constant on each of the components of the interior of $A$. Thus the component containing $B$ maps to $\left\{x^{\prime}\right\}$.

Suppose the points of $U$ in the other component map to $y^{\prime} \neq x^{\prime} . S^{3}$ is covered by 2-spheres through $x_{1}, y_{4}$. All of these 2-spheres except one intersect both components of the interior of $A$. Assume $y^{\prime}$ is not on the circle 
( $\left.x^{\prime}, x_{1}^{\prime}, y_{4}^{\prime}\right)$-otherwise, interchange $x_{1}$ and $x_{2}$. This means all of $U$ maps into the union of the two 2-spheres, $S^{\prime}$ and $\left(x^{\prime}, x_{1}^{\prime}, x_{2}^{\prime}, y_{4}^{\prime}\right)$.

Assume $y_{1}^{\prime}$ does not lie on the sphere $\left(x^{\prime}, x_{1}^{\prime}, x_{2}^{\prime}, y_{4}^{\prime}\right)$. (Otherwise, choose $y_{2}^{\prime}$ or $y_{3}^{\prime}$.) By the argument of the preceding paragraph, interchanging $y_{1}^{\prime}$ and $y_{4}^{\prime}$, all of $U$ maps to the union of the two 2-spheres $S^{\prime}$ and $\left(x^{\prime}, x_{1}^{\prime}, x_{2}^{\prime}, y_{1}^{\prime}\right)$. Combined with the above information, this means the image of $f$ is contained in a 2-sphere together with at most two additional points. This contradicts our assumptions on $f$.

On the other hand, suppose the entire interior of $A \cap U$ maps to $\left\{x^{\prime}\right\}$. Consider the sphere $S$ through $y_{1}, y_{2}, y_{3}, y_{4}$. Since the circle $\left(x_{1}^{\prime}, x_{2}^{\prime}, y_{4}^{\prime}\right)$ does not lie in $S^{\prime}, S \cap A$ cannot contain the circle $\left(x_{1}, x_{2}, y_{4}\right)$. Hence $S$ must intersect the interior of $A$. This contradicts the fact that $x^{\prime} \notin S^{\prime}$. The proof of the lemma is now complete.

LEMMA 2.5. If $S$ is a 2-sphere that intersects $U$, then $f(S \cap U)$ contains at least five points.

Proof. Suppose $S$ is a 2-sphere and $f(S \cap U)=\left\{x_{1}^{\prime}, x_{2}^{\prime}, x_{3}^{\prime}, x_{4}^{\prime}\right\}$. In each of the two components of the complement of $S$, choose a point of $U$ such that the two images are distinct and disjoint from $f(S \cap U)$. Call these two points $y_{1}$ and $y_{2}$. Every point in $S^{3}$ lies on a circle through $y_{1}, y_{2}$ and a point of $S$. Thus $f(U)$ is contained in the union of the four circles $C_{i}=\left(x_{i}^{\prime}, y_{1}^{\prime}, y_{2}^{\prime}\right)$, $i=1,2,3,4$. (Actually two or more of these circles may be identical.) The points $y_{1}$ and $y_{2}$ always can be chosen so that for some $k$, there is $z \in U \cap$ $C_{k}$ so that $z$ is separated from $y_{1}$ by $S$ and $z^{\prime} \notin\left\{x_{1}^{\prime}, x_{2}^{\prime}, x_{3}^{\prime}, x_{4}^{\prime}, y_{1}^{\prime}, y_{2}^{\prime}\right\}$. Replacing $y_{2}$ in the foregoing by $z$, we see that $f(U)$ is again contained in the union of four circles, $D_{i}, i=1,2,3,4$. (Again, two or more may be identical.) The intersection of these two unions is $C_{k} \cup\left\{x_{i}^{\prime}\right\}_{i \neq k}$. But clearly $f(U)$ cannot be contained in such a set.

LEMMA 2.6. There is a 2-sphere $S$ in $U$ upon which $f$ is a bijection of 2-spheres.

Proof. By the Six Point Theorem, it suffices to produce a 2-sphere $S \subseteq U$ whose image contains six points, no five of which lie on a circle.

We first observe that there is a 2-sphere in $U$ whose image is not contained in a circle. If not, choose three image points $x^{\prime}, y^{\prime}, z^{\prime} . S^{3}$ is covered by 2 -spheres that contain $x, y, z$. Hence the image of $f$ would be contained in the circle through $x^{\prime}, y^{\prime}, z^{\prime}$. This is a contradiction of our conditions on $f$.

So let us assume $S \subseteq U$ is a 2-sphere whose image is not contained in a circle. By Lemma 2.5 its image contains at least five points. To prove Lemma 2.6 we will assume the image of no 2-sphere in $U$ contains six points, no five of which lie on a circle. We will also assume $C^{\prime}$ is a circle, $f(S) \subseteq C^{\prime} \cup\left\{x^{\prime}\right\}$, 
and then argue to a contradiction using Lemma 2.2.

Denote by $S^{\prime}$ the 2-sphere determined by $C^{\prime}$ and $x^{\prime}$. By Lemma 2.1, $f(S) \subseteq S^{\prime}$. Since $f(S) \supseteq\{5$ pts $\}, C^{\prime} \cap f(S) \supseteq\{4$ pts $\}$. Choose $x_{1}^{\prime}, x_{2}^{\prime}, x_{3}^{\prime}$, $x_{4}^{\prime} \in C^{\prime} \cap f(S)$. We claim there are two image points $y_{1}^{\prime}, y_{2}^{\prime} \in U-S^{\prime}$ such that $y_{2}^{\prime}$ does not lie on the circle containing $x_{1}^{\prime}, x_{2}^{\prime}, y_{1}^{\prime}$, and the 2-sphere $S_{1}^{\prime}$ determined by $x_{1}^{\prime}, x_{2}^{\prime}, y_{1}^{\prime}, y_{2}^{\prime}$ does not contain $x^{\prime}$.

If the six points $x_{1}^{\prime}, x_{2}^{\prime}, x_{3}^{\prime}, x_{4}^{\prime}, y_{1}^{\prime}, y_{2}^{\prime}$ lie on a 2-sphere then the 2-sphere $\left(x_{1}, x_{2}, x_{3}, y_{1}\right)$ maps to this 2-sphere, contradicting our assumption.

Thus $\left\{x_{1}^{\prime}, x_{2}^{\prime}, x_{3}^{\prime}, x_{4}^{\prime}, y_{1}^{\prime}, y_{2}^{\prime}\right\}$ does not lie on a 2-sphere. So $y_{1}^{\prime}, y_{2}^{\prime}$ and two points of $\left\{x_{1}^{\prime}, x_{2}^{\prime}, x_{3}^{\prime}, x_{4}^{\prime}\right\}$ determine a 2-sphere. Assuming $x^{\prime}$ lies on each of these 2-spheres, we have a manifest contradiction. Suppose $x^{\prime} \notin S_{1}^{\prime}=$ $\left(x_{1}^{\prime}, x_{2}^{\prime}, y_{1}^{\prime}, y_{2}^{\prime}\right)$.

Let $S_{1}$ be the 2-sphere $\left(x_{1}, x_{2}, y_{1}, y_{2}\right)$. Note that $x \notin S_{1}$. Let $C_{1}=S \cap S_{1}$. So $f\left(C_{1}\right)=\left\{x_{1}^{\prime}, x_{2}^{\prime}\right\}$.

Let $R$ be a connected open set on $S$. If $f(R) \subseteq\left\{x_{1}^{\prime}, x_{2}^{\prime}\right\}$, then $f$ must be constant on that portion of the circle $\left(x, x_{3}, w\right), w \in R$, which meets $R$. Thus $f$ is constant on such $R$.

Consider the components of $S-C_{1}$. The image of one of these components must contain $x^{\prime}$. The image of the other component cannot be $\left\{x_{1}^{\prime}, x_{2}^{\prime}\right\}$ by the above paragraph. Thus we may assume the image of this other component contains $x_{3}^{\prime}$.

Now the family of circles $\left(x, x_{3}, z\right), z \in C_{1}$, covers $S$ and maps to the union of the two circles $\left(x^{\prime}, x_{1}^{\prime}, x_{3}^{\prime}\right)$ and $\left(x^{\prime}, x_{2}^{\prime}, x_{3}^{\prime}\right)$. This union does not contain $x_{4}^{\prime}$. This contradiction establishes the lemma.

We are now able to complete the proof of Theorem 2. Let $S$ be the 2-sphere guaranteed by Lemma 2.6. For image points $x^{\prime}, y^{\prime} \notin f(S)$, let $S_{1}$ be a 2-sphere through $x, y$ and a circle on $S$. Since $f\left(S_{1} \cap U\right)$ contains a circle and two points off this circle, the Six Point Theorem tells us $f$ is a bijection on $S_{1}$. Therefore $f$ is $1-1$ on $U$.

Let $B$ be an open ball in $U$. Since $f$ is 1-1 on $U$ and preserves 2-spheres (Lemma 2.1), we know by Theorem 1 that the image of $B$ is also an open ball. It is also true that $f^{-1}$ is circle-preserving (Lemmas 1.6 or 1.8). Therefore, $f$ is a 2-sphere preserving homeomorphism of an open set of $S^{3}$. Moebius' theorem [6] concludes that $f$ must be the restriction to $U$ of an element of the inversive group of the 3-sphere. This completes the proof of Theorem 2.

IV. The $n$-dimensional case. The next theorem shows that every circlepreserving function of an $n$-sphere $S^{n}$ whose image in $S^{n+m}$ contains at least $2 n+2$ points suitably spread about is a bijection of $n$-spheres that preserves $(n-1)$-spheres. The statement of this theorem is global although our methods allow us to deduce a local theorem also. However, the local theorem 
is not as neatly stated and, for clarity, we have separated the two.

TheOREM 3 (The $2 n+2$ TheOREM). Suppose (1) $n$ and $m$ are integers, $n>2$ and $m \geqslant 0$, (2) $f$ is a function from the sphere $S^{n}$ to the sphere $S^{n+m}$, (3) $f$ sends every circle into a circle (not assumed to be unique), (4) $S_{i}$ is an $i$-sphere for $i=2, \ldots, n$, (5) $S_{2} \subseteq \cdots \subseteq S_{n}$, (6) $f\left(S_{2}\right)$ contains (at least) six points such that no circle contains more than four of these six points, and $(7) f\left(S_{i+1}\right)-$ $f\left(S_{i}\right)$ contains at least two image points for $i=2, \ldots, n-1$. Then $f$ is 1-1, onto an $n$-sphere, and preserves $(n-1)$-spheres; that is, if we identify $f\left(S^{n}\right)$ and $S^{n}$ (by a similarity), then $f$ is an element of the inversive group of $S^{n}$.

Proof. The restriction of $f$ to $S_{2}$ is an element of the inversive group of $S^{2}$ by the global version of the Six Point Theorem (i.e., the case $U=S^{2}$ ).

Assume $f$ is $1-1$ on $S_{i}, 2 \leqslant i \leqslant k$. Let $x_{1}^{\prime}$ and $x_{2}^{\prime}$ be image points in $f\left(S_{k+1}\right)-f\left(S_{k}\right)$. (These points are guaranteed by hypothesis (7).) If $x$, $y \in S_{k+1}-S_{k}$ and $x^{\prime}=y^{\prime} \neq x_{1}^{\prime}$, consider a 2-sphere through $x, y, x_{1}$ and a circle on $S_{k}$. The restriction of $f$ to this 2-sphere satisfies the hypotheses of the Six Point Theorem. Therefore it could not have been true that $x^{\prime}=y^{\prime}$. Thus $f$ is 1-1 on $S_{k+1}$, in particular, $f$ is 1-1 on $S^{n}$.

In order to show $f$ preserves $k$-spheres, $2 \leqslant k \leqslant n-1$, we assume it preserves $(k-1)$-spheres. Let $S$ be a $k$-sphere and $S_{0}$ a $(k-1)$-sphere in $S$. If $x \in S-S_{0}$, then $S$ is the union of all circles through $x$ and two points of $S_{0}$. Thus $f(S)$ is contained in a $k$-sphere. Clearly $f(S)$ is onto a $k$-sphere, and, in particular, $f$ is onto an $n$-sphere. The proof is complete.

We now state the local version of this theorem. Its proof is almost identical except the full (i.e., local) Six Point Theorem must be invoked in the appropriate places instead of the global version.

THEOREM 3'. Suppose the following conditions are satisfied.

(1) $n$ and $m$ are integers, $n \geqslant 2$, and $m \geqslant 0$.

(2) $S_{i}$ is an $i$-sphere, $i=2, \ldots, n$.

(3) $S_{2} \subseteq \cdots \subseteq S_{n}$.

(4) $U_{2}$ is an open subset of $S_{2}$.

(5) $U_{i}$ is an open subset of a 2-sphere in $S_{i}, i=3, \ldots, n$.

(6) $U=\cup_{i=2}^{n} U_{i}$.

(7) $f$ is a function from $U$ into $S^{n+m}$.

(8) If $C$ is a circle in $S^{n}$, then $f(C \cap U)$ is contained in a (not necessarily unique) circle in $S^{n+m}$.

(9) $f\left(U_{2}\right)$ contains (at least) six points and no circle contains more than four of these six points.

(10) $f\left(U_{i+1}\right)-f\left(S_{i} \cap U\right)$ contains (at least) two points.

Then the image of $f$ is a subspace of an $n$-sphere and $f$ is the restriction to $U$ of an element of the inversive group of $S^{n}$. 
Remark 1. The $n=3$ case of Theorems 3 and $3^{\prime}$ is another 3-dimensional Eight Point Theorem. But, as we have partially pointed out in §III, it differs in several ways from Theorem 2. It is a stronger theorem in the sense that (i) complements of 2 -spheres are only required to contain two image points and (ii) the domain need only be the union of two spherical surfaces. Theorem 2 , on the other hand, requires (i) complements of 2-spheres to contain three image points, and (ii) the domain to be open in the topology of $S^{3}$.

Theorem 2 is stronger than the $n=3$ case of Theorems 3 and $3^{\prime}$ in the sense that the image of no (portion of a) 2-sphere is required to contain six points no five of which lie on a circle. The $n=3$ case of Theorems 3 and 3', on the other hand, has a hypothesis (viz. (9) in Theorem $3^{\prime}$ ) that immediately implies (via Theorem 1) that the image of a certain 2-sphere contains six points, no five of which lie on a circle. It is an open question to what extent theorems like Theorem 2 exist for $n$-spheres, $n \geqslant 4$.

RemarK 2. Instead of invoking the Six Point Theorem in the first paragraph of the proofs of Theorems 3 and $3^{\prime}$, it is clearly possible to change in an obvious way the hypotheses of the theorems in order to use, instead, the Eight Point Theorem. The change would require there exist a 3-sphere in $S^{n}$ whose image contains eight points, no six of which lie on a 2-sphere.

As we have remarked following Theorem 1, the possibility of a 2-dimensional Five Point Theorem exists. Such a theorem would mean only $5+2(n$ $-2)=2 n+1$ image points need be hypothesized in Theorems 3 and $3^{\prime}$. This is the best lower bound as the following example demonstrates.

EXAMPLE. Suppose $S$ is a fixed 2-sphere in the 3-sphere $S^{3}$ and $p$ is a point of $S^{3}$ off $S$. Let $f$ fix $S$ (point-wise) and map the complement of $S$ onto $p$. Then $f$ is a 2-sphere preserving map of $S^{3}$. It is, of course, not an element of the inversive group. This example shows that two additional points must be added to the image of $f$ with each unit increase in the dimension of $S^{n}$.

\section{REFERENCES}

1. J. D. Aczel and M. A. McKiernan, On the characterization of plane projective and complex Moebius-transformations, Math. Nachr. 33 (1967), 315-337.

2. C. Caratheodory, The most general transformation of plane regions which transform circles into circles, Bull. Amer. Math. Soc. 43 (1937), 573-579.

3. H. S. M. Coxeter, Introduction to geometry, 2nd ed., Wiley, New York, 1969.

4. J. Gibbons and C. Webb, Circle-preserving transformations, Notices Amer. Math. Soc. 22 (1975), Abstract \#A-682.

5. H. Schwerdfeger, Geometry of complex numbers, Univ. of Toronto Press, Toronto, Canada, 1962.

6. M. Spivak, A comprehensive introduction to differential geometry. III, IV, Publish or Perish, Boston, 1975.

Department of Economics, Illinois Institute of Technology, Chicago, Illinois 60616

Department of Mathematics, Chicago State University, Chicago, Illinois 60628 